\title{
Signal-dependent noise for b-modulation NFT-based transmission
}

\author{
Anastasiia Vasylchenkova, Maryna Pankratova, Jaroslaw Prilepsky, Nikolay Chichkov, Sergei Turitsyn \\ Aston Institute of Photonic Technologies, Aston University, B4 7ET Birmingham, UK
}

The nonlinear Fourier transform (NFT) is a potentially promising way to mitigate nonlinear signal distortions in fiber-optic communication systems [1-4]. Recently, the new approach named the $b$-modulation was introduced [1] that allows to control the temporal duration of NFT-generated signals. In the "traditional" NFT-based communication, the nonlinear spectrum, $r(\xi)$ is used to modulate data. Then the signal duration depends on the signal energy and is not predetermined and controlled. The utilisation of the NFT scattering coefficient $b(\xi)$ gives a possibility to control the pulse duration in time domain under condition that the Fourier spectrum of $b(\xi)$ is localized. In particular, the OFDM format satisfies that condition. It was already shown that the properties of the effective noise in the NFT domain differ from a circular Gaussian noise. More specifically, such a noise is signal-dependent and non-circular [2,3]. It was shown in [4] that the covariance $B_{1}$ and pseudo-covariance $B_{2}$ characteristics related to $b$-coefficient of NFT spectrum depend on the propagation distance. Here, we extend our previous work and examine noise characteristics for a novel $b$-modulation transmission introduced in [1]. We demonstrate that the variances behaviour is generic and do not depend on a specifics of NFT modulation. The presence of amplifier spontaneous emission in the optical link translates into the effective noise in the nonlinear spectral domain as an effective additive term $N(\xi, b(\xi, 0)): e^{-4 \xi^{2} L} b(\xi, L)=b(\xi, 0)+N(\xi, b(\xi, 0), L)$, where $\xi$ is the "nonlinear frequency" (NFT spectral parameter):

$$
\left\langle N(\xi) N^{*}\left(\xi^{\prime}\right)\right\rangle=2 D L \pi \delta\left(\xi-\xi^{\prime}\right) B_{1}(\xi, L),\left\langle N(\xi) N\left(\xi^{\prime}\right)\right\rangle=2 D L \pi \delta\left(\xi-\xi^{\prime}\right) B_{2}(\xi, L)
$$

for distance $L$, noise intensity $D ; B_{1,2}$ are the covariance and pseudo-covariance of the noise-corrupted field $b(\xi)$.

In this work we model the propagation of optical signal down the NFT-based single-mode fiber link (modelled through the nonlinear Schrodinger equation with additive noise) for distances up to $1000 \mathrm{~km}$. The schematics of the communication system is given in Fig. 1(a). Fig. 1(b) shows the dependence of $B_{1}\left(\xi, \xi^{\prime}\right)$ at $1000 \mathrm{~km}$, and Fig. 1(c) presents an accumulated noise intensity in NFT domain $\left\langle B_{1,2}\right\rangle$ as a function of link length. Each point in the Fig. 1(b) was obtained by averaging over 120 realizations of amplifiers noise.

(a)
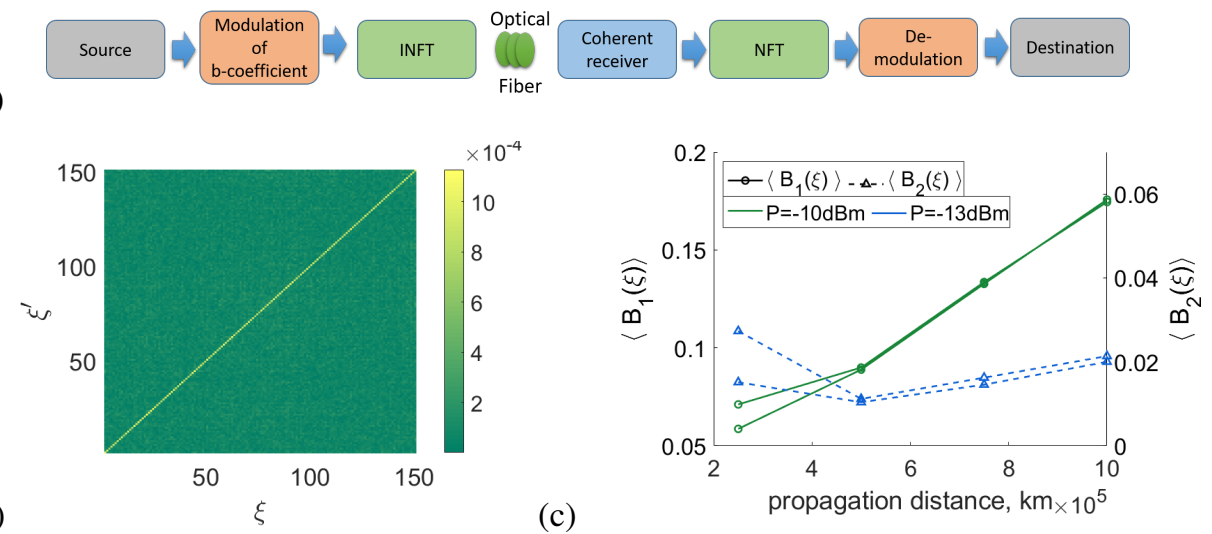

Fig. 1 The scheme of $b$-modulated NFT-based optical transmission system (a). Covariance matrix $B_{1}$ for $1000 \mathrm{~km}$ and $256 \mathrm{channels}$ (b). The dependence of covariance and pseudo-covarince matrix on propagation distance for two various values of signal power (c).

We observe that diagonal elements are noticeably higher, the correlations rapidly decreased with the increase of $\left|\xi-\xi^{\prime}\right|$, see Fig. 1 (b). However, for a pseudo-covariance matrix, we noticed that the diagonal elements were blurred for the set of transmission parameters chosen, and much less recognisable against the background. The number of OFDM channels for our plotting Fig.1 (c) was optimally selected for each distance (from 250 to 1000 $\mathrm{km}$ we used from 64 to 256 channels). It can be seen that for short distances pseudo-covariance decreases before slightly increasing again. At the same time covariance increases with propagation distance.

\section{References}

[1] S. Wahls, "Generation of Time-Limited Signals in the Nonlinear Fourier Domain via b-Modulation," 2017 European Conference on Optical Communication (ECOC), Gothenburg, paper W.3.C.6 (2017).

[2] S. A. Derevyanko, J. E. Prilepsky, and S. K. Turitsyn, "Capacity estimates for optical transmission based on the nonlinear Fourier transform," Nature Communications, 7, 12710, (2016)

[3] V. Aref, S.T. Le, and H. Buelow, Modulation over Nonlinear Fourier Spectrum: Continuous and Discrete Spectrum, J. Lightwave Technol., Vol. 36, no. 6, p. 1289 (2018).

[4] M. Pankratova, et al, "Properties of the effective noise in the nonlinear Fourier transform-based transmission," in Frontiers in Optics / Laser Science, OSA Technical Digest (Optical Society of America, 2018), paper JW3A.83 (2018). 